CLINICAL STUDY

\title{
Neuroactive steroid-serotonergic interaction: responses to an intravenous L-tryptophan challenge in women with premenstrual syndrome
}

\author{
Natalie Rasgon, Mariangela Serra ${ }^{2}$, Giovanni Biggio ${ }^{2}$, Maria Giuseppina Pisu ${ }^{2}$, Lynn Fairbanks, Sahrab Tanavoli \\ and Andrea Rapkin ${ }^{1}$ \\ Departments of Psychiatry and Biobehavioral Sciences, Neuropsychiatric Institute and ${ }^{1}$ Obstetrics and Gynecology, UCLA School of Medicine, \\ Los Angeles, California 90095, USA and ${ }^{2}$ Department of Experimental Biology, University of Cagliari, Italy \\ (Correspondence should be addressed to A Rapkin, Department of Obstetrics and Gynecology, UCLA School of Medicine, Los Angeles, \\ Calfornia 90095-1740, USA; Email: arapkin@mednet.ucla.edu)
}

\begin{abstract}
Objective: To evaluate the circulating concentrations of the neuroactive steroids in response to an i.v. L-tryptophan (L-TP) challenge across the menstrual cycle in women with premenstrual syndrome (PMS) and in controls.

Method: An i.v. L-TP challenge was administered eight times during 1 month to five women with prospectively documented PMS and five age- and body mass-matched controls. Progesterone, allopregnanolone pregnenolone and $3 \alpha-5 \alpha$-tetrahydrocorticosterone were assessed 15 and 0 min before, and at 30,60 and $90 \mathrm{~min}$ after the challenge, across the menstrual cycle.

Results: In response to L-TP challenge, only allopregnanolone concentrations were significantly increased across the cycle and this increase was of a greater magnitude in women with PMS. Pregnenolone and $3 \alpha-5 \alpha$-tetrahydrocorticosterone concentrations were not affected in women with PMS or controls after L-TP challenge.

Conclusions: The data provide evidence for possible interaction between the serotonergic system and the neuroactive steroid, allopregnanolone. Women with PMS demonstrated a more significant increase in allopregnanolone concentrations in response to L-TP challenge, which could be due to an initial low basal serotonergic tone in the luteal phase in the PMS group.
\end{abstract}

European Journal of Endocrinology 145 25-33

\section{Introduction}

Serotonergic dysfunction as a potential cause of premenstrual syndrome (PMS) has been postulated on the basis of decreased concentrations of whole-blood serotonin and diminished platelet uptake of serotonin $(1,2)$. This concept has been supported by studies demonstrating the effectiveness of selective serotonin reuptake inhibitors (SSRIs) in the treatment of premenstrual dysphoric disorder (PMDD), a severe form of PMS (3, 4). It has been speculated that SSRIs ameliorate PMS symptoms by increasing the central serotonergic tone. However, the exact mechanism is still not known, as the anxiolytic effect of SSRIs in premenstrual syndrome cannot be fully explained on the basis of augmented serotonergic transmission alone (5). As the symptoms of PMS are maximal in the late luteal phase, it has been postulated that allopregnanolone, a neuroactive anxiolytic metabolite of progesterone, may be decreased in women with PMS and might account for some of the symptoms of PMS, in particular the irritability, anxiety and tension associated with the syndrome $(6,7)$. In both animal and clinical studies it has been demonstrated that neuroactive steroids display anxiolytic, anticonvulsant and mood stabilizing effects (8-12). Behavioral effects of neuroactive steroids are mainly determined by their modulation of the $\gamma$-aminobutyric acid $\left(\mathrm{GABA}_{\mathrm{A}}\right)$ receptor complex. Progesterone, the progesterone metabolite $3 \alpha-5 \alpha-$ tetrahydroprogesterone (allopregnanolone), and the 11-deoxycorticosterone metabolite $3 \alpha-5 \alpha$-tetrahydrocorticosterone (THDOC) are positive allosteric modulators of the $\mathrm{GABA}_{\mathrm{A}}$ receptor, whereas pregnenolone sulfate is a negative allosteric $\mathrm{GABA}_{\mathrm{A}}$ receptor modulator (9). These neuroactive steroids are produced in the periphery (ovary, adrenals) and de novo in the brain (neurosteroids) either from cholesterol or through metabolism of blood-borne precursors (10).

It has been suggested that some of the effects of SSRIs on mood and seizure threshold could result from increased allopregnanolone production. Recent studies have suggested that the administration of SSRIs 
increase allopregnanolone concentrations most probably as a result of a direct effect on the enzyme $3 \alpha$-hydroxysteroid oxidoreductase, one of the two enzymes involved in the conversion of progesterone to allopregnanolone $(13,14)$.

The present study was therefore undertaken to assess whether alterations in central serotonergic tone modulate, by some mechanism, the circulating concentration of allopregnanolone in addition to the progesterone precursor pregnenolone in women with PMS. This was indirectly assessed by evaluating the plasma allopregnanolone and pregnenolone concentrations before and after the administration of L-tryptophan (L-TP), a precursor of serotonin that readily crosses the blood-brain barrier.

We evaluated allopregnanolone and pregnenolone concentration before and after L-TP challenge, twice per week for 1 month in women with prospectively documented PMS, and in controls. THDOC is predominantly an adrenal-derived metabolite of progesterone and corticosterone which has been shown to increase in the brain and plasma after stress (15-17). The preand post-challenge concentrations of THDOC were therefore also assessed in order to determine whether the changes, if any, in neuroactive steroids were specifically modulated by L-TP or were due to an increased response caused by stress.

\section{Methods}

\section{Selection of participants}

Women were recruited through advertisements in local newspapers. The study was approved by the Human Subject Protection Committee at the University of California, Los Angeles. All gave informed consent their participation. All prospective participants were evaluated for 2 months before acceptance into the study. During evaluation, the women were required to complete a daily symptom diary (18). This premenstrual diary consists of 11 of the more commonly experienced symptoms of PMS, including depression, anxiety, mood swings, irritability or anger, headaches, breast pain, low energy, edema, food cravings, avoidance of social activity, impaired relationships, and diminished work performance. Each symptom was rated on a scale of 1 (not at all) to 6 (extreme).

Eligibility criteria for the PMS group included: age 18-40 years, history of regular menses with moderate to severe premenstrual symptoms lasting for at least 1 week before menses occurring for at least the previous 6 months, negative medical history and no use of hormonal preparations including oral contraceptives for at least 6 months before enrollment into the study. All women were screened for the absence of significant medical illness (current or in the past 2 years) through history and physical examination. The body mass index
(BMI) for all participants was calculated as the weight in kilograms divided by the square of the height in meters. Women with a BMI exceeding 25 were excluded.

All the women were administered a comprehensive psychiatric evaluation, the Structured Clinical Interview for DSM-IV (19), in the follicular phase of the menstrual cycle to confirm the absence of significant current or recent (past 2 years) major psychiatric illness including substance and alcohol abuse. Those with PMS who failed to demonstrate moderate to severe symptom scores on the daily dairy with at least a $50 \%$ increase in a minimum of five symptoms including one psychological symptom between the follicular and luteal phases were excluded. Women accepted for study were required to meet the DSM-IV criteria for premenstrual dysphoric disorder. None of the women recruited into the study was receiving psychoactive medications, hormonal preparations (including oral contraceptives and thyroid replacement), mineral supplements or vitamins within the 3 months before or during the study. Eligibility criteria for controls were the same, except that premenstrual symptoms were absent. Once accepted into the study, all the women were required to complete the symptom diary for the ensuing 1 month of the study.

\section{Procedure}

The duration of the challenge study was 1 month. All women were required to abstain from alcohol and to follow a tyramine-free diet for 2 weeks before the challenge phase and throughout the study. All were required to abstain from food intake for the $12 \mathrm{~h}$ preceding the test.

Participants were required to present to the Clinical Research Center (CRC) at the UCLA Medical Center twice a week for four consecutive weeks. They began the month-long study within a few days of signing a consent form, irrespective of the day of their last menstrual period and were encouraged to keep their visits on the same day of the week (e.g. ThursdaySunday, Tuesday-Friday etc.), to eliminate fluctuations in the schedule.

Because actual visits to the CRC were not timed to the particular phase of the menstrual cycle data were transformed according to the idealized 28-day menstrual cycle. The day of luteinizing hormone $(\mathrm{LH})$ surge as determined by urinary LH detection test was considered as day 0 . The follicular phase (inclusive of mid-to-late follicular phases) was considered as ovulation days -12 to -5 , the ovulatory phase was calculated as ovulation days -4 to +3 , and the luteal phase (inclusive of mid-to-late luteal phases) was calculated as ovulation days +7 to +14 . The phase of the menstrual cycle in which blood was obtained was confirmed retrospectively by the date of onset of the next menstrual period. 
Seventy-five women were screened for the study: 28 with premenstrual syndrome and 47 control individuals. Of the 28 women with premenstrual symptomatology, 18 were excluded for one or more of the following reasons: fear of venepuncture, lack of time, unwillingness to comply with diet and alcohol restrictions for the 6-week period, premenstrual exacerbation of underlying mood and anxiety disorder, or comorbidity with substance abuse. Three women moved out of the area at the time of the beginning of the challenge phase, and one patient developed fever at the time of the first CRC visit. Of the 47 controls, 22 rejected the study because of time constraints or fear of venepuncture, seven women had irregular menses, three had a psychiatric condition other than PMS, six women did not agree to use non-steroidal contraception, and one woman had an anovulatory cycle. After the above exclusions, six women with PMS and seven controls entered the challenge phase of the study. One woman with PMS and two controls withdrew from the study after the first visit secondary to lack of interest, thus five subjects in each group completed the study.

\section{Neuroendocrine challenge}

L-Tryptophan challenge was conducted as described by Price et al. (20). On the test day all women arrived at the $\mathrm{CRC}$ at $0700 \mathrm{~h}$ after an overnight fast and remained fasting until the end of the test. Beginning at $0700 \mathrm{~h}$, the women rested on their beds supine with their heads slightly elevated, until the completion of the test (approximately $4 \mathrm{~h}$ later). They were allowed to ambulate to the bathroom, but not permitted to sleep. At $0730 \mathrm{~h}$ an i.v. catheter was placed in the forearm vein and was kept open with a flush of heparin. At $0900 \mathrm{~h}$ the women received an i.v. solution of L-TP (Arginomoto USA, Inc.) (7.0 g tryptophan diluted in $500 \mathrm{ml} 0.47 \%$ normal saline) given at a constant rate for a 20-min period. At the same time, while the women were in a sitting position, pulse and blood pressure were measured in the usual clinical fashion. Samples were placed in EDTA-treated tubes at $15 \mathrm{~min}$ before and just before (time 0) the start of the tryptophan infusion, and at 30,60 and 90 min after the start of the infusion.

\section{Biochemical methods}

The L-TP infusions were prepared by dissolving $8.4 \mathrm{~g}$ L-TP in $600 \mathrm{ml} 0.45 \%$ saline solution, with $50 \%$ sodium hydroxide added to bring the solution to $\mathrm{pH}$ 7.4. Each $600-\mathrm{ml}$ aliquot was sterilized by a passage through a $0.22-\mathrm{mm}$ filter (Millipore) and was tested for pyrogenicity and sterility before use. Plasma samples were frozen and kept at $-70{ }^{\circ} \mathrm{C}$ until required for assay.

\section{Extraction and assay of steroids}

The plasma samples $(1 \mathrm{ml})$ were diluted with $2 \mathrm{ml}$ water and then extracted three times with $3 \mathrm{ml}$ ethyl acetate and the combined organic phases were dried under vacuum. The residue was dissolved in $3 \mathrm{ml}$ $n$-hexane $(70 \%)$ and propanol $(30 \%)$. Steroids were quantified by RIA as previously described by Purdy et al. (15), with specific antibodies to pregnenolone and progesterone (ICN, Costa Mesa, CA, USA). Antibodies to allotetrahydroxycorticosterone (THDOC) and allopregnanolone were raised in rabbits and sheep and characterized as previously described (15). All measured variables were expressed in $\mathrm{ng} / \mathrm{ml}$. The limit of detection of the RIAs expressed as the minimal amount of steroids distinguishable from the zero sample was $0.01 \mathrm{ng}$ and the intra- and interassay coefficients of variation ranged between 5 and $7 \%$ and between 9 and $11 \%$ respectively.

\section{Statistical analysis}

Diary data were evaluated by non-parametric statistics using the Mann-Whitney U test (21). The progesterone, pregnenolone, allopregnanolone, and THDOC responses to L-TP challenge were assessed during the follicular, ovulatory and luteal phases of the menstrual cycle. Pre-challenge concentrations were measured and the maximum (delta) change in concentrations of each variable was calculated by subtracting the mean prechallenge value from the peak value observed at one of the 30-90 min time points in each cycle phase for each woman. The effects of PMS diagnosis on neurosteroid concentrations and delta concentrations were then assessed using multivariate analysis of variance with diagnostic group (PMS/control) as a between-group factor and cycle phase (follicular/ ovulatory/luteal) as a within-indvidual factor. Cyclephase effects were assessed for linear and quadratic components. Missing values for one cycle phase were replaced by the group mean for two individuals. For patients with more than one test in a given cycle phase, data were averaged to provide one score per woman per cycle phase. A square-root transformation was applied to correct for violation of independence of means and variances for the allopregnanolone change scores. Five women with PMS and four controls had complete data for progesterone, pregnenolone, allopregnanolone and THDOC.

All results are reported as significant when $P$ was less than or equal to 0.05 .

\section{Results}

\section{Characteristics of the participants}

The age range was from 20-35 years for both groups. Mean age for women with PMS was $24.4 \pm 0.99$ years 
Table 1 Daily diary symptom scores in women with PMS and controls in the follicular and luteal phases of the cycle. Values are means \pm S.D. (range).

\begin{tabular}{|c|c|c|c|c|}
\hline \multirow[b]{2}{*}{ Symptom } & \multicolumn{2}{|c|}{ PMS group } & \multicolumn{2}{|c|}{ Control group } \\
\hline & Follicular & Luteal & Follicular & Luteal \\
\hline Avoid social activity & $1.4 \pm 0.8(1.0-2.8)$ & $2.8 \pm 1.5(1.8-5.0)^{\star}$ & $1.0 \pm 1.0(1.0-1.2)$ & $1.1 \pm 0.2(1.0-1.4)$ \\
\hline Decreased interest & $1.6 \pm 1.0(1.0-3.4)$ & $3.1 \pm 1.5(1.6-5.0)^{\star}$ & $1.1 \pm 0.3(1.0-1.6)$ & $1.2 \pm 0.5(1.0-2.0)$ \\
\hline Edema/weight gain & $1.2 \pm 0.3(1.0-1.6)$ & $3.6 \pm 1.1(2.6-5.0)^{\star}$ & $1.1 \pm 0.1(1.0-1.2)$ & $1.4 \pm 0.8(1.0-2.8)$ \\
\hline Depression & $1.7 \pm 1.0(1.0-3.4)$ & $3.2 \pm 1.4(1.8-5.2)^{\star *}$ & $1.0 \pm 0.0(1.0-1.0)$ & $1.1 \pm 2.0(1.0-1.4)$ \\
\hline Anxiety & $1.7 \pm 1.6(1.0-4.6)$ & $2.8 \pm 1.4(1.2-4.4)^{\star \star}$ & $1.2 \pm 0.5(1.0-2.0)$ & $1.0 \pm 1.0(1.0-1.2)$ \\
\hline Mood swings & $1.6 \pm 0.9(1.0-3.0)$ & $3.2 \pm 1.3(1.8-4.6)^{\star *}$ & $1.1 \pm 0.2(1.0-2.0)$ & $1.0 \pm 1.0(1.0-1.2)$ \\
\hline Irritability & $1.4 \pm 0.9(1.0-2.4)$ & $3.0 \pm 1.4(1.4-4.8)^{\star \star}$ & $1.0 \pm 0.0(1.0-1.0)$ & $1.0 \pm 0.0(1.0-1.0)$ \\
\hline Increased appetite/cravings & $1.3 \pm 0.5(1.0-2.2)$ & $3.5 \pm 1.2(1.8-5.0)^{*}$ & $1.1 \pm 0.1(1.0-1.3)$ & $1.5 \pm 0.9(1.0-3.0)$ \\
\hline Low energy/fatigue & $2.2 \pm 1.7(1.0-4.6)$ & $3.2 \pm 1.2(2.0-5.0)^{\star \star}$ & $1.2 \pm 0.3(1.0-1.6)$ & $1.1 \pm 0.2(1.0-1.5)$ \\
\hline Headaches & $1.8 \pm 1.7(1.0-4.8)$ & $2.5 \pm 1.3(1.0-4.2)^{\star}$ & $1.2 \pm 0.4(1.0-2.0)$ & $1.0 \pm 0.0(1.0-1.0)$ \\
\hline Breast pain & $1.1 \pm 0.1(1.0-1.2)$ & $2.6 \pm 1.5(1.0-4.4)$ & $1.1 \pm 0.2(1.0-1.4)$ & $1.0 \pm 0.0(1.0-1.0)$ \\
\hline Sleep disturbance & $1.5 \pm 0.5(1.0-2.0)$ & $2.1 \pm 1.2(1.0-3.8)$ & $1.0 \pm 0.0(1.0-1.0)$ & $1.0 \pm 0.0(1.0-1.0)$ \\
\hline Difficulty concentrating & $1.6 \pm 1.4(1.0-4.2)$ & $3.0 \pm 1.6(1.0-5.0)$ & $1.4 \pm 0.7(1.0-2.6)$ & $1.5 \pm 0.9(1.0-2.6)$ \\
\hline
\end{tabular}

${ }^{\star} P<0.05,{ }^{\star \star} P<0.01$, PMS compared with control group.

and for controls it was $27.0 \pm 6.7$ years. All study participants were unmarried and nulliparous. There were no significant differences in the mean ages or BMI between groups. All women reported regular menstrual cycles ranging from 23 to 33 days in duration, and all ovulated as determined by the urinary LH detection kits.

Table 1 shows the mean symptom scores for the PMS and control groups during the follicular (ovulation days -12 to -5 ) and luteal (ovulation days +7 to +14 ) phases. Luteal phase symptoms scores in the PMS group were significantly greater than those in controls for most symptoms (Table 1). None of these comparisons differed significantly in the follicular phase.

\section{Hormonal variables}

Pre-challenge hormonal changes As all the women started the L-TP infusion in the follicular phase, the
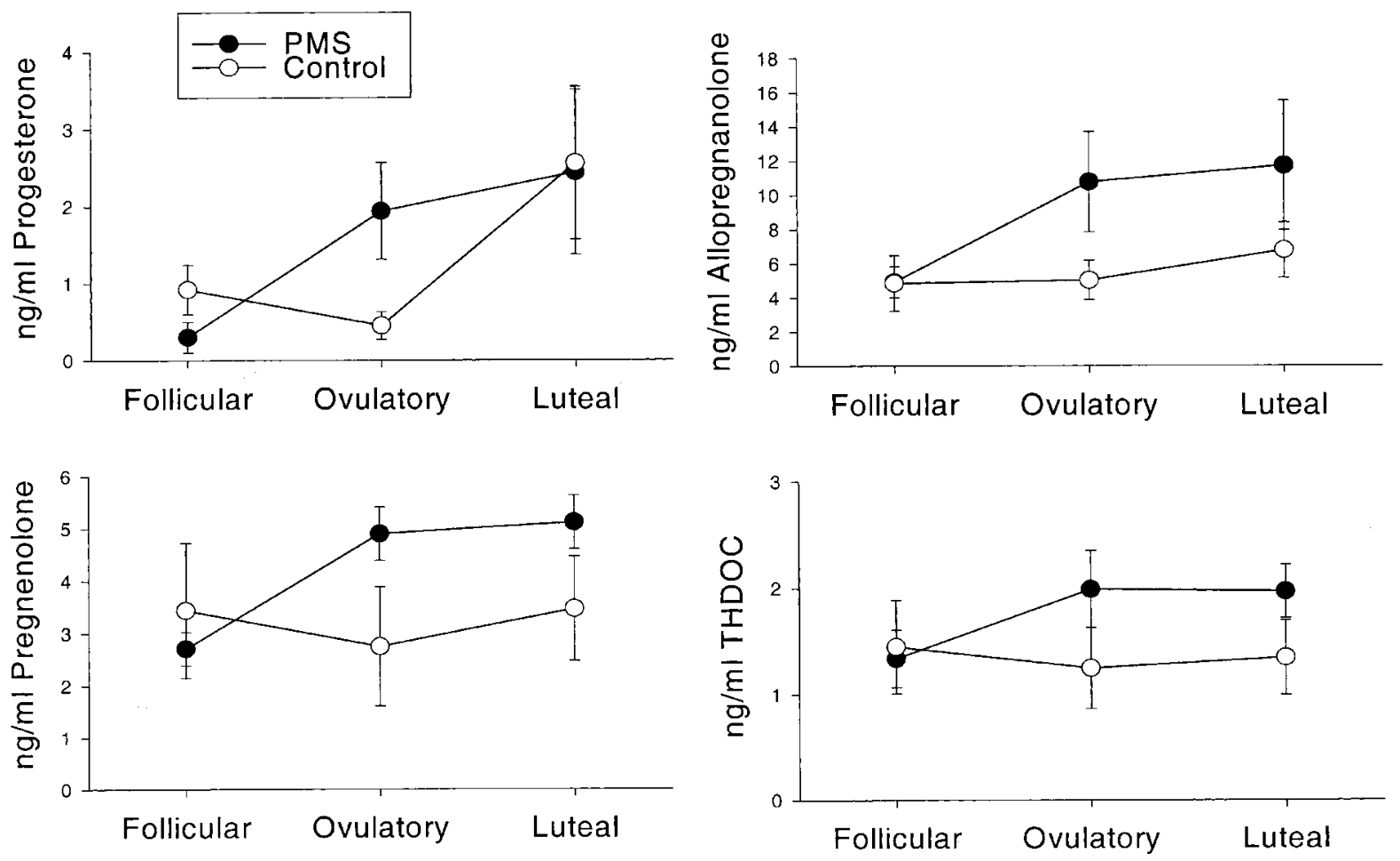

Figure 1 Mean ( \pm S.E.) pre-challenge progesterone, allopregnanolone, pregnenolone and THDOC for women with PMS and controls, by cycle phase.

www.eje.org 
ovulatory and luteal phase pre-challenge samples in these phases cannot be considered 'basal', because the prior L-TP infusions may have influenced the prechallenge concentrations of neuroactive steroids. As expected, pre-challenge progesterone, pregnenolone and allopregnanolone concentrations increased from the follicular to the ovulatory to the luteal phase both in women with PMS and in controls. Figure 1 shows the mean ( \pm S.E.) concentrations of progesterone, allopregnanolone, pregnenolone and THDOC by cycle phase for women with PMS and controls. There were significant interactions between group and cycle phase in the analyses of variance for each of the four steroids (progesterone: $\mathrm{F}=5.70, P<0.05$; allopregnanolone: $\mathrm{F}=4.15, \quad P=0.05 ;$ pregnenolone: $\mathrm{F}=8.32, \quad \mathrm{P}<$ 0.05; THDOC: $\mathrm{F}=6.77, P<0.05)$. This indicates that women with PMS differ from normal controls in the pattern of change in neuroactive steroids over the menstrual cycle.

Allopregnanolone concentrations increased only slightly across cycle phases for the controls (4.85 \pm 3.2 compared with $5.0 \pm 2.3$ and $6.7 \pm 3.2 \mathrm{ng} / \mathrm{ml}$ ), but more than doubled from the follicular (4.9 \pm $2.0 \mathrm{ng} / \mathrm{ml})$ to the ovulatory $(10.8 \pm 6.6 \mathrm{ng} / \mathrm{ml})$ and luteal phases $(11.7 \pm 3.8 \mathrm{ng} / \mathrm{ml})$ for those with PMS. The increase in allopregnanolone from the follicular to the luteal phase was statistically significant for the PMS group (paired $\mathrm{t}=4.23, P=0.01$ ) but not for the controls.

Similar to allopregnanolone, pre-challenge concentrations of pregnenolone remained relatively constant throughout the cycle for the control group $(3.44 \pm 2.6$ compared with $2.75 \pm 2.3$ and $3.46 \pm 2.0 \mathrm{ng} / \mathrm{ml}$ ) from the follicular to the ovulatory and luteal phases. In contrast, in women with PMS a significant increase was found in the pre-challenge pregnenolone concentrations from follicular to the luteal phase of the menstrual cycle $(2.71 \pm 0.73$ compared with $4.89 \pm$ 1.13 and $5.12 \pm 1.14 \mathrm{ng} / \mathrm{ml}$; paired $\mathrm{t}=3.61, P<$ $0.05)$.

THDOC did not change throughout the menstrual cycle for the control group (1.45 \pm 0.88 compared with $1.25 \pm 0.75$ and $1.35 \pm 0.69 \mathrm{ng} / \mathrm{ml})$, but increased across the cycle in the PMS group (Fig. 1). Pre-challenge THDOC concentrations increased by approximately $50 \%$ from the follicular (1.34 \pm $0.60 \mathrm{ng} / \mathrm{ml})$ to the luteal $(1.96 \pm 0.56 \mathrm{ng} / \mathrm{ml})$ phases for the women with PMS (paired $\mathrm{t}=5.28, P<0.01$ ).

Response to L-TP challenge Figure 2 shows the mean $( \pm$ S.E.) change in allopregnanolone, pregnenolone and THDOC concentrations after L-TP challenge for women with PMS and controls. The PMS group had a greater increase in allopregnanolone in response to the
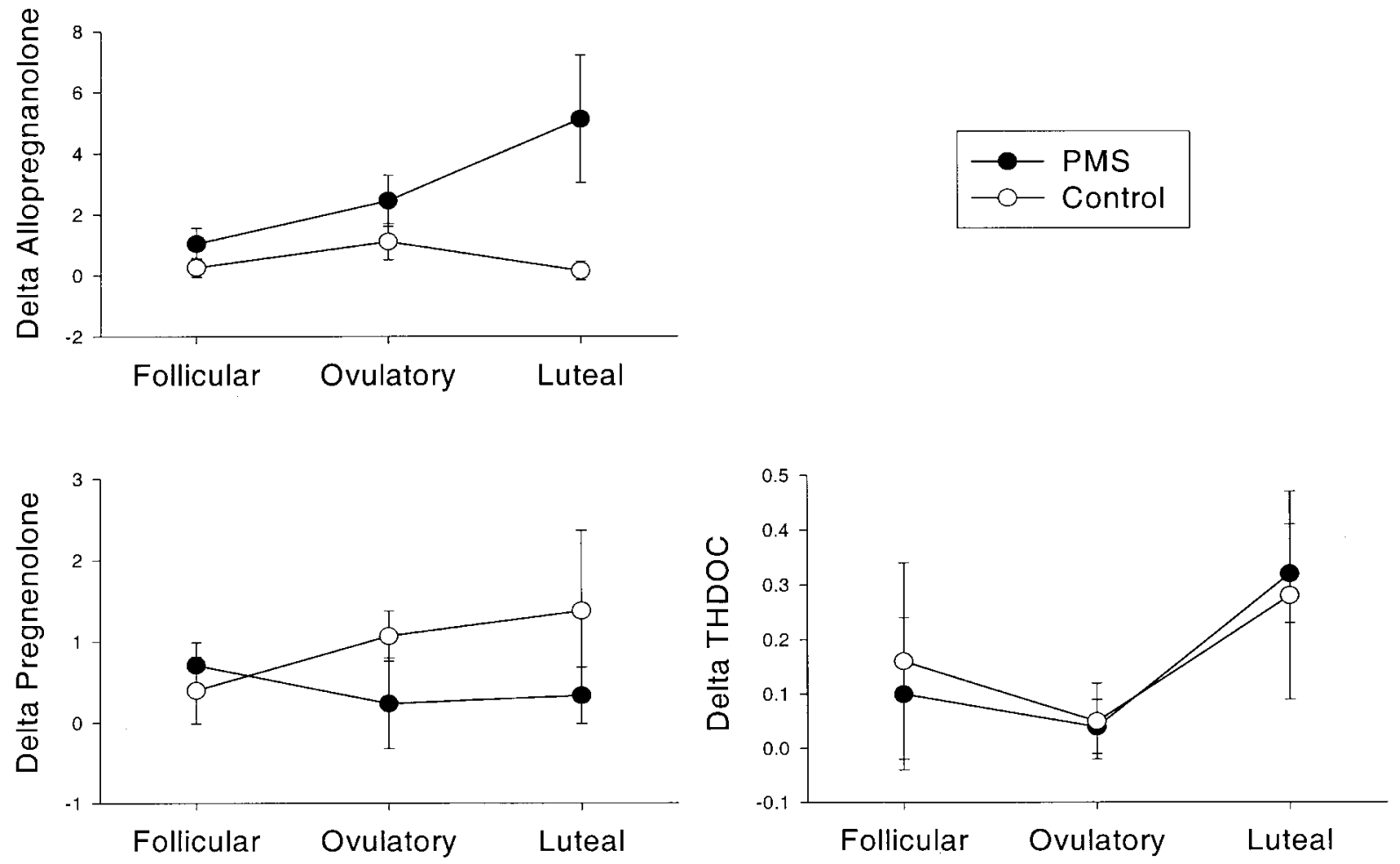

Figure 2 Mean ( \pm S.E.) maximum change (Delta) in allopregnanolone, pregnenolone and THDOC after L-TP challenge for women with PMS and controls, by cycle phase. 
challenge compared with the control group, particularly during the luteal phase (PMS compared with control: $\mathrm{F}=5.53, P=0.05$; group by cycle phase interaction: $F=5.79, P<0.05)$. These differences were not observed in either the delta pregnenolone or THDOC responses following L-TP challenge (Fig. 2).

\section{Discussion}

Although numerous hypotheses have been advanced to explain the etiology of PMS, the cause of the syndrome is still unknown. We have previously demonstrated that whole-blood serotonin is decreased in the luteal phase in women with PMS compared with controls (1). On this basis, we speculated that the decrease in wholeblood serotonin may potentially reflect a decrease in serotonin concentration in the brain and may thereby lead to a decrease in central serotonergic activity, which may account for some of the symptoms of PMS. Subsequently other investigators have demonstrated decreased platelet uptake of serotonin in women with PMS (2) and there are numerous reports that administration of SSRIs and other serotonergic agonists leads to improvement in the symptoms of PMS/PMDD (3, 4, $22,23)$. More recently, a relationship between the administration of SSRIs and the concentration of the neurosteroid allopregnanolone in human plasma and cerebrospinal fluid (CSF), and in rat brain sections (13, 24-26) have been reported. Allopregnanolone is a positive allosteric modulator of the $\mathrm{GABA}_{\mathrm{A}}$ receptor and it has been demonstrated by some $(6,7)$, but not by others (27-29), that a luteal phase decrease in plasma allopregnanolone concentration is associated with PMS. Others reports have suggested that there is a decrease in the GABAergic sensitivity to neurosteroid administration in the luteal phase in women with PMS compared with controls $(30,31)$. Moreover, other receptors coupled to ion channels such as glycine receptors, nicotine acetylcholine receptors and 5-hydroxytryptamine 3 receptors have been identified as potential target for a modulation by various neuroactive steroids (32).

The primary objective of the present study therefore, was to assess whether i.v. challenge with the serotonergic precursor L-TP led to an increase in peripherally measured neuroactive steroid concentrations, with specific focus on allopregnanolone. We also wanted to assess whether the changes in allopregnanolone concentrations after L-TP challenge were different in women with PMS than in controls. As expected, we observed an increase in pre-challenge progesterone and allopregnanolone concentrations in the luteal phase compared with the follicular phase both in women with PMS and controls. In this respect our findings are consistent with those of other studies $(27,28,33)$, indicating that changes in progesterone concentrations, which are greater in the luteal phase, and peripheral allopregnanolone concentrations parallel each other. In a previous study we had reported that, in the luteal phase, basal allopregnanolone concentrations were lower in women with PMS than in controls. In this study, as L-TP infusion was started randomly during the cycle, the luteal phase concentrations of allopregnanolone and other measured neuroactive steroids cannot be considered to be basal values as, on retrospective analysis, these women had already received L-TP infusion on at least three prior occasions, and two reported symptom improvement in the luteal phase of the challenge month. Alternatively, women with PMS may have been more stressed than controls, and allopregnanolone is known to increase after stress $(15,16)$. Higher levels of perceived stress and physiological arousal in women with PMS has been shown in response to activities of daily living and experimental stress paradigms (34-36). The greater luteal phase pre-challenge concentrations of THDOC in the PMS group compared with that in controls also suggest that the PMS patients may have been more stressed at the time of the pre-challenge plasma sampling.

The main finding in this study was that, after L-TP challenge, circulating allopregnanolone concentrations were significantly increased in both controls and women with PMS during the luteal phase compared with the follicular phase of the menstrual cycle. As peripheral allopregnanolone concentrations parallel the progesterone concentrations, it is not surprising that, after L-TP challenge, allopregnanolone concentrations increased significantly only in the luteal phase when its substrate progesterone was also increased.

This increase in allopregnanolone concentrations in the luteal phase after the administration of L-TP in both controls and women with PMS strongly suggest that there is a relationship between central serotonergic activity and circulating allopregnanolone concentrations. The greater increase in allopregnanolone concentrations after administration of L-TP in women with PMS compared with controls most probably reflects the lower baseline serotonergic activity in this group of women, as has been suggested by us and others (1, 2, $37,38)$. Therefore any mechanism by which central serotonergic activity is increased, as with the administration of L-TP in our study, or after administration of SSRIs - especially if the baseline central serotonergic activity is low - is likely to increase allopregnanolone concentrations to a greater degree than when the central serotonergic activity is high. This may potentially explain and, at least in part, account for some of the benefit observed in women with PMS treated with serotonergic agonists such as SSRIs, L-TP, fenfluramine and serotonergic tricyclic antidepressants $(3,4,22,23$, 37, 39).

Another plausible explanation for the greater concentrations of the anxiolytic neurosteroid allopregnanolone, both before and after L-TP challenge in the setting of PMS symptomatology, is a reduced 
GABAergic sensitivity in women with PMS. Several in vitro studies have demonstrated that even after shortterm exposure to neurosteroids, cultured hippocampal and cortical cells manifest decreased GABA Argic sensitivity to allopregnanolone (40). This effect may be more pronounced in women with PMS. Sundstrom et al. (41) noted that women with PMS exhibited less sedation and less decrease in saccadic eye velocity (SEV) compared with controls in response to i.v. diazepam, midazolam or pregnanolone - all positive allosteric modulators of $\mathrm{GABA}_{\mathrm{A}}(30,41)$. Similar hyposensitivity has also been described in patients with panic disorder $(42,43)$. Panic disorder and PMS share some common symptoms and both are successfully treated with SSRIs $(3,44)$. As it has been demonstrated that allopregnanolone can induce transcription via progesterone receptors after intracellular oxidation into $5 \alpha$-dihydroprogesterone (32), the possibility of modulation, by a genomic mechanism, in the expression of $\mathrm{GABA}_{\mathrm{A}}$ receptor subunit genes, leading to a decreased sensitivity, cannot be ruled out.

THDOC is another metabolite of progesterone and corticosterone, which is produced in the periphery in the adrenal gland. After acute stress, the circulating concentration of this hormone, in addition to those of pregnenolone and allopregnanolone, are increased (15-17). Serotonin increases ACTH secretion and this could in part represent a mechanism whereby allopregnanolone derived from the adrenal gland could increase after L-TP challenge (45). However, in our study, after L-TP administration the circulating concentration of allopregnanolone but not of THDOC or pregnenolone was increased. This strongly indicates that the increase in allopregnanolone concentration after L-TP administration in the current study was probably due to some specific effect of L-TP, rather than due to non-specific effects of acute stress.

This study is a preliminary report and has certain limitations, therefore the results should therefore be interpreted with caution. In this study we have measured peripheral circulating concentrations of neuroactive steroids and assumed a relationship with the concentration of the hormones in the brain. However some, but not all, studies in rodents do indicate some interrelationship between peripheral concentrations of these hormones, especially progesterone and allopregnanolone, with those in the brain $(46-48)$.

Our study did not examine the mechanism by which L-TP administration led to an increase in the circulating concentrations of allopregnanolone. It has been postulated by other investigators that the SSRIs fluoxetine and paroxetine increase allopregnanolone concentrations by a complex interaction of these compounds with $3 \alpha$-hydroxysteroid oxidoreductase, one of the two enzymes involved in the metabolism of progesterone to allopregnanolone, thus modulating the activity of this enzyme. Authors of another study, however, concluded that the anticonvulsant action of fluoxetine was due to enhanced synaptic action of endogenous 5-hydroxytryptamine (49). Our present findings also suggest the possibility that the effects on allopregnanolone observed with SSRIs may be at least in part due to enhanced central serogeneric activity, and not solely due to specific interaction of the SSRI with the $3 \alpha$-hydroxysteroid oxidoreductase enzyme.

In conclusion, our studies indicate for the first time a positive relationship between the administration of L-TP and plasma allopregnanolone concentrations. This is most probably due to an increase in central serotonergic tone and may potentially explain, at least in part, some of the effects observed in women with PMS treated with serotonergic agonists. Further studies are in progress, measuring the neurosteroid concentrations in CSF and correlating them with those in the peripheral circulation in women with PMS and controls and determining whether these concentrations change after modulation of central serotonergic tone.

\section{Acknowledgement}

This study was supported in part by NIH grant M01-RR00865-25.

\section{References}

1 Rapkin AJ, Edelmuth E, Chuong LC, Reading A, McGuire MT \& $\mathrm{Su}$ TP. Whole-blood serotonin in premenstrual syndrome. Obstetrics and Gynecology 1987 70 533-537.

2 Taylor DL, Mathew RJ, Ho BT \& Weinman ML. Serotonin levels and platelet uptake during premenstrual tension. Neuropsychobiology $19841216-18$.

3 Steiner M, Steinberg S, Stewart S, Carter D, Berger C, Reid R et al. Fluoxetine in the treatment of premenstrual dysphoria. New England Journal of Medicine 1995332 1529-1534.

4 Yonkers KA, Halbreich U, Freeman E, Brown C, Endicott J, Frank E et al. Symptomatic improvement of premenstrual dysphoric disorder with sertraline treatment. Journal of the American Medical Association 1997 278 983-988.

5 Guidotti A \& Costa E. Can the antidysphoric and anxiolytic profiles of selective serotonin reuptake inhibitors be related to their ability to increase brain 3alpha-5alpha-tetrahydroprogesterone (allopregnanolone) availability? Biological Psychiatry 1998 44 865-873.

6 Rapkin AJ, Morgan M, Goldman L \& Manesh VB. Progesterone metabolite allopregnanolone in women with premenstrual syndrome. Obstetrics and Gynecology $1997 \mathbf{9 0}$ 709-714.

7 Monteleone P, Luisi S, Tonetti A, Bernardi F, Genazzani AD, Luisi M et al. Allopregnanolone concentrations and premenstrual syndrome. European Journal of Endocrinology 2000142 269273.

8 Majewska MD. Neurosteroids: endogenous bimodal modulators of the $\mathrm{GABA}_{\mathrm{A}}$ receptor. Mechanism of action and physiological significance. Progress in Neurobiology 199238 379-395.

9 Paul SM \& Purdy RH. Neuroactive steroids. Federation of American Societies of Experimental Biology Journal 6 2311-2322. 
10 Purdy RH, Morrow AL, Blinn JR \& Paul SM. Synthesis, metabolism, and pharmacological activity of 3-alpha-hydroxysteroids which potentiate GABA receptor mediated chloride ion uptake in rat cerebral cortical synaptosomes. Journal of Medical Chemistry 199033 1572-1581.

11 Rupprecht R, Strohle A, Herman B, Michele FD, Spalletta G, Pasini A et al. Neuroactive steroid concentrations following metyrapone administration in depressed patients and healthy volunteers. Biological Psychiatry 199844 912-914.

12 Karavolas HJ \& Hodges D. Neuroendocrine metabolism of progesterone and related progestin steroids and neuronal activity. In Ciba Foundation Symposium 153, pp 22-55. Chichester: John Wiley \& Sons, 1990.

13 Uzunov DP, Cooper TB, Costa E \& Guidotti A. Fluoxetine-elicited changes in brain neurosteroid content measured by negative ion mass fragmentography. PNAS 199693 12599-12604.

14 Griffin LD \& Mellon SH. Selective serotonin reuptake inhibitors directly alter activity of neurosteroidogenic enzymes. PNAS 1999 96 13512-13517.

15 Purdy RH, Morrow AL, Moore PH \& Paul SM. Stressinduced elevations of the $\gamma$-aminobutyric acid type A receptor-active steroids in the rat brain. PNAS $1991 \mathbf{8 8}$ 4553-4557.

16 Barbaccia ML, Roscetti G \& Bolacchi F. Stress-induced increase in brain neuroactive steroids: antagonism by abercanil. Pharmacology and Biochemistry of Behavior $1996 \mathbf{5 4} 205-210$.

17 Barbaccia ML, Concas A, Serra M \& Biggio G. Stress and neurosteroids in adult and aged rats. Experimental Gerontology $199833697-712$.

18 Endicott J, Nee J, Cohen J \& Halbreich H. Premenstrual changes patterns and correlates of daily rating. Journal of Affective Disorders 198610 127-135.

19 Diagnostic and Statistical Manual of Mental Disorders, edn 2, Washington D.C.: APA Press, 1994.

20 Price LH, Charney DD, Delgado PL \& Heininger GR. Serotonin function and depression: neuroendocrine and mood responses to intravenous L-tryptophan in depressed patients and healthy comparison subjects. American Journal of Psychiatry $1991 \mathbf{1 4 8}$ 1518-1525.

21 Siegel S. Nonparametric Statistics for the Behavioral Sciences. New York: McGraw Hill, 1956.

22 Steinberg S, Annable L, Young SN \& Liyanage N. A placebocontrolled clinical trial of L-tryptophan in premenstrual dysphoria. Biological Psychiatry 199945 313-320.

23 Brzezinski AA, Wurtman JJ, Wurtman RJ, Gleason R, Greenfield J \& Nader T. D-Fenflurramine suppresses the increased calorie and carbohydrate intakes and improves the mood of women with premenstrual depression. Obstetrics and Gynecology $1990 \mathbf{7 6}$ 296-301.

24 Uzunova V, Sheline Y, Davis JM, Rasmusson A, Uzunov DP, Costa $\mathrm{E}$ et al. Increase in the cerebrospinal fluid content of neurosteroids in patients with unipolar major depression who are receiving fluoxetine or fluvoxamine. PNAS $1998 \mathbf{9 5}$ 3239-3244.

25 Romeo E, Strohle A, Spalletta G, di Michele F, Hermann B, Holsboer $\mathrm{F}$ et al. Effects of antidepressant treatment on neuroactive steroids in major depression. American Journal of Psychiatry 1998155 910-913.

26 Strohle A, Romeo E, Hermann B, Pasini A, Spalletta G, De Michelle $\mathrm{F}$ et al. Concentrations of 3 alpha-reduced neuroactive steroids and their precursors in plasma of patients with major depression and after clinical recovery. Biological Psychiatry 1999 45 274-277.

27 Schmidt PJ, Purdy RH, Moore PH, Paul SM \& Rubinow DR. Circulating levels of anxiolytic steroids in the luteal phase in women with premenstrual syndrome and in control subjects. Journal of Clinical Endocrinology and Metabolism $1994 \mathbf{7 9}$ 1256-1260.

28 Wang MD, Seippel L, Purdy RH \& Backstrom T. Relationship between symptom severity and steroid variation in women with premenstrual syndrome: study on serum pregnenolone, pregnenolone sulfate, $5 \alpha$-pregnan-3, 20-dione and $3 \alpha$-hydroxy- $5 \alpha /$ pregnan-20-one. Journal of Clinical Endocrinology and Metabolism 199681 1076-1082.

29 Bicikova M, Dibbelt L, Hill M, Hamph R \& Stark L. Allopregnanolone in women with premenstrual syndrome. Hormone Metabolism Research $199830227-230$.

30 Sundstrom I, Andersson A, Nyberg S, Ashbrook D, Purdy RH \& Backstrom T. Patients with premenstrual syndrome have a different sensitivity to a neuroactive steroid during the menstrual cycle compared with control subjects. Neuroendocrinology 1998 67 126-138.

31 Sundstrom I \& Backstrom T. Citalopram increases pregnenolone sensitivity in patients with premenstrual syndrome: an open trial. Psychoneuroendocrinology 199823 173-188.

32 Rupprecht R \& Holsboer F. Neuroactive steroids: mechanism of action and neuropharmacological perspectives. Trends in Neurosciences 199922 410-416.

33 Genazzani AR, Petraglia F, Bernardi F, Casarosa E, Salvestroni C, Tonetti A et al. Circulating levels of allopregnanolone in humans: gender, age, and endocrine influences. Journal of Clinical Endocrinology and Metabolism $1998 \mathbf{8 3}$ 2099-2103.

34 Schmidt PJ, Grover GN \& Hoban MC. State-dependent alterations in the perception of life events in menstrualrelated mood disorders. Americn Journal of Psychiatry 1990 147 230-234.

35 Woods NF, Lentz MJ \& Mitchell ES. Arousal and stress response across the menstrual cycle in women with three perimenstrual symptom patterns. Research in Nurses Health $19941799-100$.

36 Dickson-Parnell B \& Zeichner A. The premenstrual syndrome: psychophysiologic concomitants of perceived stress and low back pain. Pain $198834161-169$.

37 Eriksson E. Serotonin reuptake inhibitors for the treatment of premenstrual dysphoria. International Clinical Psychopharmacology 1999 B14 S27-S33.

38 Halbreich MD \& Tworek H. Altered serotonergic activity in women with dysphoric premenstrual syndromes. International Journal of Psychiatry in Medicine $1993231-27$.

39 Sundblad C, Hedberg MA \& Eriksson E. Clomipramine administered during the luteal phase reduces the symptoms of premenstrual syndrome: a placebo controlled trial. Neuropsychopharmacology 9 133-145.

40 Brussaard AB, Devay P, Leyting-Vermuelen JW \& Kits KS. Changes in properties and neurosteroid regulation of GABAergic synapses in the supraoptic nucleus during the mammalian female reproductive cycle. Journal of Physiology $1999 \mathbf{5 1 6}$ 513-524.

41 Sundstrom I, Andersson A, Nyberg S, Ashbrook D, Purdy RH \& Backstrom T. Reduced benzodiazepine sensitivity in patients with premenstrual syndrome, a pilot study. Psychoneuroendocrinology 199722 25-38.

42 Roy-Burne PP, Cowley DS, Greenblatt DJ, Shader RI \& Hommer D. Reduced benzodiazepine sensitivity in panic disorder. Archives of General Psychiatry $1990 \mathbf{4 7} 534-538$.

43 Brambilla F, Bellodi L, Perna G, Biggio G \& Serra M. Plasma neurosteroid concentrations in panic disorder. Neuropsychopharmacology 200023 S24.

44 Johnson MR, Lydiard RB \& Ballenger JC. Panic disorder. Pathophysiology and drug treatment. Drugs $1995 \mathbf{4 9}$ 328-344.

45 Jorgensen H, Knigge U, Kjaer A \& Warberg J. Adrenocorticotropic hormone secretion in rats induced by stimulation with serotoninergic compounds. Journal of Neuroendocrinology $1999 \mathbf{1 1}$ 283-290.

46 Concas A, Follesa P, Barbaccia ML, Purdy RH \& Biggio G. Physiological modulation of $\mathrm{GABA}_{\mathrm{A}}$ receptor plasticity by progesterone metabolites. European Journal of Pharmacology $1999375225-235$. 
47 Barbaccia ML, Roscetti G, Trabucchi M, Purdy RH, Mostallino MC, Concas A et al. The effects of inhibitors of GABAergic transmission and stress on brain and plasma allopregnanolone concentrations. British Journal of Pharmacology $19971201582-1588$.

48 Follesa P, Serra M, Cagetti E, Giuseppina P, Porta S, Floris S et al. Allopregnanolone synthesis in cerebellar granule cells: roles in regulation of $\mathrm{GABA}_{\mathrm{A}}$ receptor expression and function during progesterone treatment and withdrawal. Molecular Pharmacology 200057 1262-1270.
49 Pasini A, Tortorella A \& Gale K. The anticonvulsant action of fluoxetine in substantia nigra is dependent upon endogenous serotonin. Brain Research $1996 \mathbf{7 2 4} 84-88$.

Received 5 September 2000

Accepted 2 February 2001 